REVIEW SERIES

\title{
Cough - 6: Which investigations are most useful in the diagnosis of chronic cough?
}

\section{P A McGarvey}

Thorax 2004;59:342-346. doi: 10.1136/thx.2004.021832

There is no consensus as to the best diagnostic strategy for chronic cough. Many protocols combine empirical trials of treatment with laboratory investigations. More precise diagnostic tools and improved therapeutic options are required. Until then, the management of chronic cough will remain a clinical challenge.

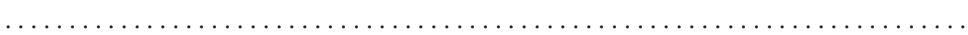

Correspondence to:

Dr L McGarveyDepartment of Medicine, Institute of Clinical Science, The Queen's University of Belfast, Grosvenor Road, Belfast BT12 6BJ, UK; l.mcgarvey@qub.ac.uk

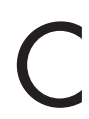

ough remains the most common reason for patients to seek medical attention. ${ }^{1}$ In the last 30 years there have been considerable advances in our understanding of the mechanisms and management of cough. ${ }^{2}$ Basic science has combined closely with clinical practice and the pharmaceutical industry to develop new diagnostic and therapeutic strategies. Currently, self-medication with over the counter antitussives probably remains the best approach for a cough associated with upper respiratory tract infection, despite doubts as to their efficacy. ${ }^{3}$ However, the management of chronic cough remains a challenge for the clinician.

Chronic cough as the only symptom can account for $10 \%$ of all referrals made to general respiratory clinics. ${ }^{4}$ The treatment success in such clinics with no established diagnostic protocol is poor, contrasting with the excellent treatment responses reported by specialist cough clinics. ${ }^{5-7}$ However, a careful review of reports detailing experience from a number of well established cough clinics has indicated some variance in diagnostic approach and treatment success. ${ }^{8-13}$

A number of factors may account for the discrepancy between units and include differences in how diagnoses are established and some variation in the study populations. In both North America and Europe guidelines have now been drawn up and recommendations made on the management of chronic cough. ${ }^{14}{ }^{15}$ Despite this, no completely satisfactory agreement on the clinical assessment and treatment of cough exists. The main problem centres on provision of guidelines, which consider the availability of diagnostic tests to the general and specialist physician in both hospital and general practice.

Recently, questions have arisen over the cost effectiveness of diagnostic protocols which involve an elaborate series of laboratory investigations. ${ }^{16}$ A number of recent studies have suggested that trials of empirical treatment for chronic cough offered a more cost beneficial option than laboratory testing. ${ }^{16}{ }^{17}$ It is therefore timely to review the investigations currently available, to highlight those which are most useful, and to consider the future role of new tools in the evaluation of cough.

\section{DIAGNOSTIC STRATEGIES FOR CHRONIC COUGH}

Chronic cough has been defined as one lasting more than 3 weeks, ${ }^{18}$ although a cough following an upper respiratory tract infection may persist for considerably longer. It is therefore more satisfactory to define chronic cough as one lasting more than 8 weeks and to recognise an overlap period of 3-8 weeks.

Current diagnostic protocols for chronic cough have been based on the work of Irwin and colleagues first reported over 20 years ago. ${ }^{5}$ The foundation for this approach was an appreciation of the anatomical sites of receptors comprising the afferent limb of the human cough reflex. This approach has been termed the "anatomic diagnostic protocol". Using a combination of history, physical examination, and laboratory investigations directed at these sites, possible causes for the cough were considered. The suspected cause could then be confirmed if the cough resolved or significantly improved after a trial of diagnosis specific treatment. The main findings from the work of this group can be summarised as follows: ${ }^{5} 6$

- By following the anatomic diagnostic protocol, a cause for cough can be found in almost all cases $(>90 \%)$.

- Asthma, gastro-oesophageal reflux disease, and rhinitis are responsible for most cases of chronic cough in the non-smoker.

- Combination therapy may be required as persistent cough may have more than one cause (multiple causes in up to $40 \%$ of cases).

A number of subsequent prospective studies in both community and hospital settings using modifications of the protocol have extended these observations. ${ }^{8-13}$ The key findings are as follows:

- Systematic evaluation remains the most effective approach to chronic cough. ${ }^{8-13}$

- Asthma, postnasal drip syndrome, and gastrooesophageal reflux remain the most important causes of chronic cough to consider. ${ }^{8-13}$

- Chronic cough may be simultaneously caused by more than one condition (19-62\% of cases). ${ }^{11}{ }^{13}$

- Empirical treatment can be substituted for specific diagnostic testing. ${ }^{9}$ 
- Eosinophilic bronchitis may account for up to $15 \%$ of cough patients being referred for specialist attention. ${ }^{12}$

- Despite comprehensive evaluation and treatment, up to $20 \%$ of patients remain symptomatic. ${ }^{811}$

\section{MAKING THE DIAGNOSIS}

The most challenging patient is one who has coughed for many years, has never smoked, is not taking an angiotensin converting enzyme inhibitor, has no abnormal findings on physical examination, and has a normal chest radiograph. Diagnostic protocols are most relevant for this group of patients, although the best combination of diagnostic testing and trials of empirical treatment is unknown.

\section{History and examination}

A careful history and thorough physical examination remains the first step in the evaluation of a patient with chronic cough. It is unlikely that any single feature at this stage is likely to clinch the diagnosis. Reliance on the characteristics and associated symptoms of the cough can be misleadingthat is, symptoms of postnasal drip may reflect only coexistent rhinitis and the absence of dyspepsia does not rule out reflux as the cause of the cough. The predictive values of cough characteristics identified from the history have been calculated and are shown in table $1 .^{11}$

The finding of wheeze or crackles on chest auscultation, or inflamed and obstructed nasal passages on inspection of the upper airway will influence the subsequent investigation or choice of treatment. More commonly, though, the examination is entirely normal.

\section{CLINICAL INVESTIGATIONS}

A number of baseline investigations which should be performed routinely and a range of more complex tests often limited by availability may be requested. An overview of these is presented, with the most valuable aspects of the individual tests highlighted.

\section{Chest radiograph}

Patients referred to specialist cough clinics most commonly have a normal chest radiograph. However, review of a recent film is mandatory as any significant abnormality will alter the diagnostic algorithm and avoid unnecessary investigation.

\section{Spirometric and peak expiratory flow measurements} Baseline spirometric results are likely to be normal in patients referred for specialist opinion. Spirometry is available in most hospitals and in an increasing number of primary care clinics. When available, it should be performed with reversibility testing in all patients with chronic cough. Peak expiratory

Table 1 Predictive value of symptom characteristics obtained from history

\begin{tabular}{llll}
\hline & $\begin{array}{l}\text { No with } \\
\text { positive } \\
\text { history }\end{array}$ & $\begin{array}{l}\text { No correctly } \\
\text { identified }\end{array}$ & $\begin{array}{l}\text { Positive } \\
\text { predictive } \\
\text { value (\%) }\end{array}$ \\
\hline $\begin{array}{l}\text { Asthma (nocturnal cough, } \\
\text { precipitated by cold air, } \\
\text { exercise, aerosols) }\end{array}$ & 27 & 15 & 56 \\
$\begin{array}{l}\text { PNDS (throat clearing, sensation } \\
\text { of postnasal drip, nasal } \\
\text { discharge, previous sinusitis) } \\
\text { GORD (dyspepsia, cough worse } \\
\text { after meals) }\end{array}$ & 20 & 14 & 52 \\
\hline
\end{tabular}

PNDS = postnasal drip syndrome; GORD = gastro-oesophageal reflux disease.

Reproduced from McGarvey et al " with permission. flow (PEF) measurement in patients with persistent cough presenting in primary care has focused on the diagnosis of airflow obstruction. For this purpose it may have some merit, but the use of PEF measurements to assess bronchodilator response appears to have limitations compared with conventional measurements of forced expiratory volume in 1 second $\left(\mathrm{FEV}_{1}\right){ }^{19}$ The value of serial PEF measurements for determining diurnal variability has not been properly assessed and is infrequently requested by specialist cough clinics.

\section{Bronchoprovocation testing}

Bronchial challenge testing can provide very useful clinical information about patients with chronic cough. Virtually every diagnostic protocol for cough uses direct challenge methods (usually histamine or methacholine) and this review will be restricted to these. Bronchial hyperreactivity in a patient with cough and normal spirometric measurements raises the possibility of cough variant asthma. However, a definitive diagnosis cannot be made until the cough responds to specific asthma treatment. Airway hyperreactivity may develop during an acute viral respiratory illness and may persist for some months afterwards. ${ }^{20}$ Cough may persist for many weeks following an upper respiratory tract infection and a positive challenge test in this circumstance may be diagnostically misleading. Airway hyperreactivity to methacholine has also been found in patients with reflux oesophagitis but no respiratory complaints. ${ }^{21}$

A negative bronchial challenge effectively excludes asthma as the diagnosis but does not eliminate a cough that may respond to steroid treatment. Particular interest has focused on patients with chronic cough and an eosinophilic bronchitis but with none of the airway dysfunction typically associated with asthma. ${ }^{12} 22{ }^{23}$ A negative bronchial challenge may therefore prompt the clinician to assess the airway (most commonly using induced sputum) for evidence of an eosinophilia. Cough associated with non-asthmatic eosinophilic bronchitis has been discussed in an earlier review in this series. ${ }^{24}$

\section{Upper airway provocation studies}

Extrathoracic airway dysfunction may account for asthmalike symptoms in particular cough. Extrathoracic airway responsiveness can be assessed by recording the maximal inspiratory flow-volume curve during conventional bronchial challenge testing. ${ }^{25}$ Extrathoracic airway hyperresponsiveness in the absence of bronchial hyperresponsiveness may be an indicator of upper airway disease as a cause for cough. Using flow-volume loops, variable extrathoracic upper airway obstruction has been observed in a series of patients with cough due to postnasal drip syndrome which improved with treatment. ${ }^{26}$ Precise interpretation of extrathoracic reactivity is difficult and the test is likely to remain primarily a research tool.

\section{Sinus imaging}

The appropriate role and timing of sinus imaging in patients with chronic cough has yet to be established. It should follow ear, nose and throat inspection and may identify abnormalities not appreciated on examination. In a prospective study of patients with chronic cough, abnormalities of the plain sinus radiograph were identified in only $29 \%$ of patient diagnosed with cough due to sinusitis. ${ }^{27}$ Computed tomographic (CT) imaging of the sinuses in children with chronic cough revealed abnormalities in two thirds of cases. However, no correlation was found between any of the findings and the child's cough. ${ }^{28}$ In a prospective evaluation of a diagnostic protocol for cough which included routine CT sinus imaging, the predictive value of the scan was no better than ear, nose and throat examination in accurately identifying upper airway disease. ${ }^{11}$ 
Plain sinus radiographs therefore are of little value in the evaluation of chronic cough, and sinus CT scanning should be reserved for refractory cases which may require surgical intervention.

\section{Gastrointestinal investigations}

The association between gastro-oesophageal reflux and chronic cough has been comprehensively reviewed in a previous article in this series. ${ }^{29}$ The salient points relevant to evaluating reflux cough in the outpatient setting are presented here. An empirical trial of antireflux therapy may be the best first line approach for patients with cough and heartburn. However, as cough may be the only clinical manifestation of gastro-oesophageal reflux or if a therapeutic trial fails, laboratory investigations may be required. Barium swallow has low sensitivity and specificity and is of little value in the initial evaluation of chronic cough. ${ }^{30}$ Although not always available, 24 hour oesophageal $\mathrm{pH}$ monitoring is currently the best single test to help characterise any link between gastro-oesophageal reflux and cough. Dual probe $\mathrm{pH}$ studies are now commonly used and have extended our knowledge of the site and extent of reflux disease. A review of dual probe data in cough patients found that reflux to the proximal probe is an uncommon event, ${ }^{31}$ although reflux to the hypopharynx and beyond (laryngopharyngeal reflux) certainly does occur in some patients and may be the reason for the cough. ${ }^{32}$ However, 24 hour oesophageal pH monitoring is not without limitations and no consensus exists as to how best to define the temporal association between a cough and a reflux episode or to identify individuals most likely to benefit from antireflux therapy. There needs to be some caution in the interpretation of a negative $\mathrm{pH}$ profile. In one recent series, patients who continued to cough despite complete acid suppression as shown by oesophageal $\mathrm{pH}$ monitoring had resolution of their symptom after antireflux surgery. There are a number of explanations for this, but the most plausible is that, in some patients, non-acid refluxate may be of equal importance in the pathogenesis of cough. Up to one third of reflux episodes in patients with gastrooesophageal reflux disease are non-acid, and a promising development has been the simultaneous intraoesophageal impedance and $\mathrm{pH}$ measurement of acid and non-acid reflux events. ${ }^{33}$ This technique should differentiate better the role of non-acid refluxate in the mediation of cough and extend our understanding of the role of gastro-oesophageal reflux in the pathogenesis of many airway diseases.

\section{High resolution computed tomography (CT) of the thorax}

A high resolution CT scan may identify parenchymal lung disease not apparent on the chest radiograph. In a prospective study of patients with chronic cough, all of whom were undergoing a complex diagnostic protocol which included a high resolution CT scan of the chest, bronchiectasis was identified in nearly a quarter of cases. ${ }^{13}$ It was not clear from the study if this diagnosis could have been made from the history, examination and/or chest radiograph alone. Targeted use in patients with chest radiographic abnormalities and/or clinical findings on chest examination has a diagnostic yield of about $17 \%{ }^{34}$ In summary, there is no role for thoracic CT scanning in the routine evaluation of chronic cough.

\section{Bronchoscopy}

The diagnostic yield from bronchoscopy in the routine evaluation of chronic cough is low, about $5 \% .{ }^{14}$ This reflects the low frequency of smokers and radiograph abnormalities among patients seeking medical attention for their cough. In spite of this, bronchoscopy has significant diagnostic potential in selected patients where the more common causes have been rigorously excluded. In a review of such patients referred with refractory cough, bronchoscopic diagnoses were found in a quarter of cases and included broncholithiasis, tracheobronchopathia, and laryngeal dyskinesia. ${ }^{35}$ Aspirated foreign bodies may go unrecognised for prolonged periods of time, although bronchoscopy is probably only indicated when there is a clue in the medical history. Fibreoptic bronchoscopy provides the opportunity for airway sampling either by mucosal biopsy or bronchial lavage, although this approach has been largely replaced by non-invasive techniques.

\section{ASSESSING AIRWAY INFLAMMATION Induced sputum}

A number of groups have recently adapted the conventional diagnostic strategies for chronic cough to include induced sputum. ${ }^{12}{ }^{21}$ The key aspect has been the demonstration of an airway eosinophilia in patients without functional abnormalities, in particular bronchial hyperreactivity associated with asthma. Eosinophilic bronchitis without asthma as a cause for chronic cough was first described by Gibson et al. ${ }^{22}{ }^{36} \mathrm{~A}$ sputum eosinophil count of more than 3\% has been suggested as indicative of eosinophilic bronchitis, and the condition has now been added to the spectrum of diseases that cause chronic cough, accounting for up to $15 \%$ of cases referred for specialist attention. ${ }^{12}$ Although debate remains as to whether eosinophilic bronchitis exists as a separate diagnostic entity, ${ }^{37}$ the finding of a sputum eosinophilia has important treatment implications with high success rates following inhaled corticosteroid therapy. Ideally, induced sputum should be requested after exclusion of the other common causes and after demonstration of normal airway hyperresponsiveness (provocative concentration of methacholine or histamine producing a $20 \%$ decrease in $\mathrm{FEV}_{1}$ $\left.\left(\mathrm{PC}_{20}\right)>16 \mathrm{mg} / \mathrm{ml}\right) .^{12}$

Unfortunately, induced sputum is not widely available outside specialist centres and is unlikely to be routinely incorporated into diagnostic protocols for cough. In primary care and in general medical clinics, empirical trials of inhaled corticosteroids are still commonly used for chronic cough, which further obscures the true prevalence of eosinophilic bronchitis and its distinction from asthma.

\section{Exhaled breath}

Exhaled nitric oxide (NO) levels appear to be lower in nonasthmatic coughers, allowing some differentiation from asthmatic cough. ${ }^{38}$ Exhaled NO may represent a simpler alternative to induced sputum tests, but currently it has no clear diagnostic role in the management of chronic cough. The measurement of many different inflammatory molecules in breath condensate, although currently a research procedure, may have a place in the diagnosis of chronic cough in the future.

\section{Inhalation cough challenge}

Patients with persistent cough have a hypersensitive cough reflex to a range of tussive agents which diminishes with successful treatment. ${ }^{10}$ Citric acid and capsaicin are the two most widely used in cough challenge testing, although others include tartaric acid and ultrasonically nebulised distilled water. $^{39}{ }^{40}$ The methodology involved is similar to bronchial provocation testing but, in contrast to these inhalation tests, no agreed universal standards for cough challenge testing exist. ${ }^{41}$ The particular value of cough reflex testing is in assessing the efficacy of antitussive therapy. There are no discriminating features on cough challenge testing which are likely to be of diagnostic value, and hence its place outside a research capacity is likely to remain limited. 


\section{Cough recording}

Interest in cough monitoring has concentrated mainly on developing an objective means of measuring cough frequency and assessing severity. Differences in the characteristics of the cough sound and flow pattern between asthma, bronchitis, and interstitial fibrosis have been reported. ${ }^{42}$ Analysis of overnight cough recording determined differences in character and intensity of cough sounds between patients with cystic fibrosis and cryptogenic fibrosing alveolitis. ${ }^{43}$ These observations open the possibility of using cough monitoring as a diagnostic tool. Ambulatory devices offer the best opportunity for this, but cost and portability together with insufficient computer memory have been the major limitations. Current advances in technology should resolve these problems and widen the opportunity to record cough accurately.

\section{Psychological assessment}

Psychogenic cough can be included in the range of recognised functional respiratory disorders. In children a characteristic "honking" or barking sound is described and is often attributed to a "habit cough". However, a more complex "cough tic" disorder may underlie the problem. ${ }^{44}$

Psychogenic cough in an adult patient should only be considered following thorough exclusion of a physical cause. The value of a prompt and accurate diagnosis is manifest in the successful outcomes reported after psychotherapy. ${ }^{45}$ As cough is known to have an adverse impact on the quality of an individual's life (in particular psychosocial aspects), cough specific quality of life questionnaires have been developed. Although not diagnostic, such instruments provide a measurement of the size and nature of this effect. ${ }^{46}{ }^{47}$

\section{EMPIRICAL TREATMENT}

Empirical treatment can be thought of as a "diagnostic trial" in patients with cough. Patients reporting symptoms suggestive of gastro-oesophageal reflux or postnasal drip syndrome should first be offered a trial of empirical treatment. In a placebo controlled trial in patients with cough associated with gastro-oesophageal reflux disease, omeprazole $40 \mathrm{mg}$ daily for 8 weeks produced an effective and sustained resolution of symptoms. ${ }^{48}$ Older (first generation) antihistamines in combination with decongestants have been recommended as empirical treatment for postnasal drip syndrome. ${ }^{914}$ The diagnostic role of empirical treatment has not been thoroughly studied so the dose and duration of treatment have yet to be established. If the cough persists despite compliance with adequate dosing for a sufficient duration, laboratory investigations in that area are not likely to be helpful.

\section{CONCLUSIONS}

A systematic approach to diagnosis and treatment remains the most effective way to manage chronic cough. Important questions remain as to the complexity and cost effectiveness of existing diagnostic algorithms. The baseline investigations that should be available in all or almost all patients with persistent cough are a chest radiograph and spirometric tests. The choice of additional tests will be determined by the clinician's view of the individual case and what laboratory investigations are available. The appraisal of current diagnostic protocols has highlighted the limitations of some of the constituent investigations. In particular, while a negative bronchoprovocation test rules out asthma, it does not exclude a steroid responsive cough. Similarly, a negative 24 hour oesophageal $\mathrm{pH}$ probe suggests acid reflux is not the cause of cough, but it does not rule out non-acid reflux. Current investigations therefore need to be further refined and more emphasis should be placed on the diagnostic role of empirical trials of treatment. Future efforts should be directed at designing more reliable and precise tests. Some promising developments in the near future may well improve our diagnostic approach to this common and debilitating symptom.

\section{REFERENCES}

1 Schappert SM. National Ambulatory Medical Care Survey: 1995-96 summary. Vital Health Stat 1999;142:i-vi, 1-122.

2 Morice AH, Kastelik JA. Cough - 1: Chronic cough in adults. Thorax 2003;58:901-7.

3 Schroeder K, Fahey T. Systematic review of randomised controlled trials of over the counter cough medicines for acute cough in adults. BMJ 2002;324:329-31.

4 McGarvey L, Heaney LG, MacMahon J. A retrospective survey of diagnosis and management of patients presenting with chronic cough to a general chest clinic. Int J Clin Pract 1998;52:158-61.

5 Irwin RS, Corrao WM, Pratter MR. Chronic persistent cough in the adult: the spectrum and frequency of cases and successful outcome of specific therapy. Am Rev Respir Dis 1981;123:414-7.

6 Irwin RS, Curley FJ, French CL. Chronic cough: the spectrum and frequency of causes, key components of the diagnostic evaluation and outcome of specific therapy. Am Rev Respir Dis 1990:141:640-7.

7 Smyrnios NA, Irwin RS, Curley FJ. Chronic cough with a history of excessive sputum production: the spectrum and frequency of causes, key components of the diagnostic evaluation, and outcome of specific therapy. Chest 1995; 108:991-7.

8 Poe HR, Harder RV, Israel RH. Chronic persistent cough: experience in diagnosis and outcome using an anatomic diagnostic protocol. Chest 1989;95:723-7.

9 Pratter MR, Bartter T, Akers S, et al. An algorithmic approach to chronic cough. Ann Intern Med 1993;1 19:977-83.

10 O'Connell F, Thomas VE, Fuller RW, et al. Cough sensitivity to inhaled capsaicin decreases with successful treatment of chronic cough. Am J Respir Crit Care Med 1993;150:374-80.

11 McGarvey LPA, Heaney LG, Lawson JT, et al. Evaluation and outcome of patients with chronic non-productive cough using a comprehensive diagnostic protocol. Thorax 1998;53:738-43.

12 Brightling C, Ward R, Goh KL, et al. Eosinophilic bronchitis is an important cause of chronic cough. Am J Respir Crit Care Med 1999; 160:406-10

13 Palombini BC, Villanova CA, Araujo E, et al. A pathogenic triad in chronic cough: asthma, postnasal drip syndrome and gastrooesophageal reflux disease. Chest 1999:116:279-84.

14 Irwin RS, Boulet LP, Cloutier MM, et al. Managing cough as a defence mechanism and as a symptom. A consensus panel report of the American College of Chest Physicians. Chest 1998;114:133-81S.

15 European Task Force on Chronic Cough. Guidelines prepared by a European Respiratory Society Task Force on Chronic Cough. Eur Respir J 2004 (in press).

16 Lin L, Poh KL, Lim TK. Empirical treatment of chronic cough: a cost effective analysis. Proc AMIA Symp 2001:383-7.

17 Ours TM, Kavuru MS, Schilz RJ, et al. A prospective evaluation of esophageal testing and a double blind, randomized study of omeprazole in a diagnostic and therapeutic algorithm for chronic cough. Am J Gastroenterol 1999:94:3131-8.

18 Curley FJ, Irwin RS, Pratter MR. Cough and the common cold. Am Rev Respir Dis 1988; 138:305-11.

19 Thiadens HA, De Bock GH, Van Houwelingen JC, et al. Can peak expiratory flow measurements reliably identify the presence of obstruction and bronchodilator response as assessed by $\mathrm{FEV}_{1}$ in primary care patients presenting with a persistent cough? Thorax 1999;54:1055-60.

20 de Kluijver J, Grundberg K, Sont JK, et al. Rhinovirus infection in non asthmatic subjects: effects on intrapulmonary airways. Eur Respir $J$ 2002:20:274-9.

21 Ferrari $M$, Olivieri $M$, Sembenini $C$, et al. Tussive effect of capsaicin in patients with gastroesophageal reflux without cough. Am J Respir Crit Care Med 1995;151:557-61.

22 Gibson PG, Dolovich J, Denberg JA, et al. Chronic cough: eosinophilic bronchitis without asthma. Lancet 1989:1346-8.

23 Carney IK, Gibson PG, Murree-Allen K, et al. A systematic evaluation of mechanisms in chronic cough. Am J Respir Crit Care Med 1997:156:211-6.

24 Dicpingaitis PV. Cough - 4: Cough in asthma and eosinophilic bronchitis. Thorax 2004;59:71-2.

25 Bucca C, Rolla G, Brussino L, et al. Are asthma-like symptoms due to bronchial or extrathoracic airway dysfunction? Lancet 1995:346:791-5.

26 Irwin RS, Pratter MR, Holland PS, et al. Postnasal drip causes cough and is associated with reversible upper airway obstruction. Chest 1984;85:346-52.

27 Pratter MR, Bartter T, Lotano R. The role of sinus imaging in the treatment of chronic cough in adults. Chest 1999;116:1287-91.

28 Tatli MM, San I, Karaoglanoglu M. Paranasal sinus computed tomographic findings of children with chronic cough. Int $J$ Pediatr Otorhinolaryngol $2001 ; 60: 213-7$

29 Fontana GA, Pistolesi M. Cough - 3: Chronic cough and gastro-oesophageal reflux. Thorax 2003;58:1092-5. 
30 Richter JE, Castell DO. Gastroesophageal reflux. Pathogenesis, diagnosis, and therapy. Ann Intern Med 1982;97:93-103.

31 Irwin RS, French CL, Curley FJ, et al. Chronic cough due to gastroesophageal reflux. Clinical, diagnostic, and pathogenetic aspects. Chest 1993;104:1511-7.

32 Cohen JT, Bach KK, Postma GN, et al. Clinical manifestations of laryngopharyngeal reflux. Ear Nose Throat J 2002;81(9 Suppl 2):19-23.

33 Sifrim D, Holloway R, Silny J, et al. Acid, nonacid, and gas reflux in patients with gastroesophageal reflux disease during ambulatory 24 -hour $\mathrm{pH}$ impedance recordings. Gastroenterology 2001;120:1588-98.

34 Ojoo J, Kastelik JA, Mulrennan S, et al. Selective use of thoracic tomographs in patients with chronic cough (abstract). Eur Respir J 2002;20(Suppl 38): $449 \mathrm{~s}$.

35 Sen RP, Walsh TE. Fibreoptic bronchoscopy for refractory cough. Chest 1991;99:33-5.

36 Gibson PG, Hargreave FE, Girgis-Gabardo A, et al. Chronic cough with eosinophilic bronchitis: examination for variable airflow obstruction and response to corticosteroid. Clin Exp Allergy 1995;25:127-32.

37 McGarvey L, Heaney L, MacMahon J, et al. Eosinophilic bronchitis is an important cause of chronic cough. Am J Respir Crit Care Med $2000 ; 161: 1763-4$

38 Chatkin JM, Ansarin K, Silkoff PE, et al. Exhaled nitirc oxide as a noninvasive assessment of chronic cough. Am J Respir Crit Care Med 1999;159:1810-3.
39 Fujimura M, Sakamoto S, Kamio Y, et al. Cough receptor sensitivity and bronchial responsiveness in normal and asthmatic subjects. Eur Respir J 1992;5:291-5.

40 Fontana GA, Lavorini F, Pistolesi M. Water aerosols and cough. Pulm Pharmacol Ther 2002;15:205-11.

41 Morice AH, Kastelik JA, Thompson R. Cough challenge in the assessment of cough reflex. Br J Clin Pharmacol 2001;52:365-75.

42 Piirila P, Sovijarvi AJ. Differences in acoustic and dynamic characteristics of spontaneous cough in pulmonary diseases. Chest 1989;96:46-53.

43 Hall LE, Smith A, Earis JE, et al. Patterns of cough in cystic fibrosis and cryptogenic fibrosing alveolitis (abstract). Thorax 2001;56(Suppl III):iii71.

44 Ojoo JC, Kastelik JA, Morice AH. A boy with a disabling cough. Lancet 2003;361:674.

45 Mastrovich JD, Greenberger PA. Psychogenic cough in adults: a report of two cases and review of the literature. Allergy Asthma Proc 2002;23:27-33.

46 French CT, Irwin RS, Fletcher KE, et al. Evaluation of a cough-specific qualityof-life questionnaire. Chest 2002;121:1123-31.

47 Birring S, Prudon B, Carr AJ, et al. Development of a symptom specific health status measure for patients with chronic cough: Leicester Cough Questionnaire (LCQ). Thorax 2003;58:339-43.

48 Kiljander T, Salomaa ERM, Hietanen EK, et al. Chronic cough and gastrooesophageal reflux: a double-blind placebo-controlled study with omeprazole. Eur Respir J 2000;16:633-8.

\section{LUNG ALERT}

\section{Doubling the dose of inhaled corticosteroid for asthma exacerbations does not decrease subsequent use of systemic steroids}

$\Delta$ Harrison TW, Oborne J, Newton S, et al. Doubling the dose of inhaled corticosteroid to prevent asthma exacerbations: randomised controlled trial. Lancet 2004;363:271-5

T his blinded trial with concealed randomisation compared doubling the dose of inhaled corticosteroid for 14 days with continuing the same dose when asthma symptoms or peak flow worsened in patients monitoring themselves daily at home. The 390 patients enrolled had been given oral corticosteroid or transiently doubled their inhaled corticosteroid dose in the past year for worsening asthma, but had been stable for at least 2 weeks before entry to the study. All patients were using an inhaled corticosteroid, with most using a low or moderate dose. They were randomised to receive a study inhaler, identical in appearance to their usual steroid inhaler, which contained either further steroid or placebo. During the 1 year study period $53 \%$ of patients started their study inhaler due to worsening asthma, and $11 \%$ in the treatment group versus $12 \%$ in the placebo group started oral prednisolone because of a fall in peak flow of $>40 \%$, subjectively worsening asthma, or on advice of their general practitioner $(p=0.8)$. There was still no difference in oral steroid use when analysed among those who used the study inhaler (per protocol analysis) or among those in the subgroups on low/moderate versus high dose inhaled corticosteroid at baseline.

While this study does not lend support for the practice of patients doubling their inhaled corticosteroid for worsening asthma as part of their self-management plan, it also does not definitively refute it. Despite being a large trial, it was significantly underpowered to detect what could be a clinically important reduction in systemic steroid use because of the low event rate.

M Soth

Respirology Resident, McMaster University, Hamilton, Canada msoth@idirect.com 